\title{
Improvement of Communication Skills in Clinical Education
}

\author{
Woo Jeong $\mathrm{Kim}^{1}$ and Min Young $\mathrm{Kim}^{2}$ \\ ${ }^{1}$ Department of Emergency Medicine, School of Medicine, Jeju National University \\ ${ }^{2}$ College of Nursing, Jeju National University \\ musemy2@jejunu.ac.kr
}

\begin{abstract}
The purpose of this study was to identify the correlation between medical students' communication skills awareness and performance in clinical education and to determine means to improve communication. A total of 36 fourth-year medical students were enrolled in this study and were surveyed concerning communication skills during two clinical performance examinations. The survey included 20 items in four categories: beginning an interview (greeting, identification, introduction, attention, and chief complaint), gathering information (open question, reflection, facilitation, clarification, and summarizing), giving information (discovering the patient's understanding, empathy, easy terms, checking of understanding, and giving opportunities), and non-verbal communication (neat features, listening, eye contact, nodding, and silence). The students were educated repeatedly about their communication skills for 8 months in a clinical clerkship between two examinations. All items showed the significant correlations between communication skills awareness and performance, except for greeting and summarizing in the first examination and neat feature and silence in the second. Communication skills awareness and performance were significantly improved in terms of attention, reflection, summarizing, discovering the patient's understanding, empathy, checking understanding, and silence. This study demonstrated that medical students' awareness of communication skills was mostly correlated with performance and communication awareness and performance were improved following clinical education.
\end{abstract}

Keywords: communication skills, performance, medical education, awareness

\section{Introduction}

Communication between healthcare providers and patients in a clinical situation is essential for gathering important information, making proper decisions, and giving helpful messages. Providing education concerning patient-centered communication skills can increase patient knowledge and self-efficacy and may improve patient self-care through improved communication during the patient encounter. Further, teaching communication skills can improve biological outcomes, quality of life, and survival [1].

However, a common medical school clinical education experience consist of a situation in which they encounter the patient for the first time and they find that they do not know how to cope with the patients. They feel like they are left all alone and to try to do the best they can. So, young doctors have complained about their lack of communication training during medical school. In addition, it is well established that patients complain more often about poor communication than about inadequate treatment [2].

Most medical school and nursing colleges teach communication skills by various methods because of the importance of good clinical communication, but there are questions regarding 
how these communication skills transfer from communication training to actual patient care [3].

This study's purpose was to identify the correlation between medical students' communication skills awareness and performance and to determine communication improvement following their clinical education.

\section{Methods}

\subsection{Subjects}

The subjects were 36 fourth-year medical students, who had their first clinical performance examination (CPX) right before their fourth year and another CPX eight months later.

\subsection{Procedure}

These students reported their communication skills awareness and performance in each $\mathrm{CPX}$, according to the questionnaire's instructions. Clinical education of communication skills was performed in several clinical clerkships after the first CPX. All 36 medical students had their second CPX after eight months, when their clinical clerkship was complete. Communication skills education included lectures on core items according to the medical communication process, watching communication videos, and role playing.

\subsection{Instruments}

The medical education communication skills questionnaires consisted of 20 items in four categories: beginning the interview (greeting, identification, introduction, attention, and chief complaint), gathering information (open question, reflection, facilitation, clarification, and summarizing), giving information (discovering patient's understanding, empathy, easy terms, checking of understanding, and giving opportunities), and non-verbal communication (neat feature, listening, eye contact, nodding, and silence). The definitions and examples of the 20 items in the four categories are listed in Table 1. Each question was rated along a 5-point Likert scale, ranging from 1 (strongly disagree) to 5 (strongly agree).

Table 1. Description of Items and Definitions in Communication Skills

\begin{tabular}{|c|l|l|l|}
\hline Categories & \multicolumn{1}{|c|}{ Items } & \multicolumn{1}{|c|}{ Definitions } & \multicolumn{1}{|c|}{ Examples } \\
\hline \multirow{4}{*}{$\begin{array}{c}\text { Beginning } \\
\text { the } \\
\text { interview }\end{array}$} & Greeting & $\begin{array}{l}\text { Expressing a greeting upon } \\
\text { meeting patients. }\end{array}$ & Hello, Good morning. \\
\cline { 2 - 5 } & Introduction & $\begin{array}{l}\text { Formally making you known to } \\
\text { the patients. }\end{array}$ & $\begin{array}{l}\text { I'm Dr. Kim, one of emergency } \\
\text { physicians. }\end{array}$ \\
\cline { 2 - 5 } & Attention & $\begin{array}{l}\text { General interest that leads patients } \\
\text { to feel comfort. }\end{array}$ & I'm sorry that you waited so long. \\
\cline { 2 - 5 } & Chief complaint & $\begin{array}{l}\text { Asking patients a cause of } \\
\text { complaining. }\end{array}$ & $\begin{array}{l}\text { Could you please tell me what } \\
\text { problem brought you to hospital? }\end{array}$ \\
\hline \multirow{3}{*}{$\begin{array}{c}\text { Gathering } \\
\text { information }\end{array}$} & Open question & $\begin{array}{l}\text { Assessment of a wide range of } \\
\text { issues, concerns, or feelings. }\end{array}$ & Would you tell me more about it? \\
\cline { 2 - 5 } & Reflection & $\begin{array}{l}\text { Support by listening to the } \\
\text { patient. }\end{array}$ & Right. ; That should not be easy. \\
\hline
\end{tabular}




\begin{tabular}{|c|c|c|c|}
\hline & Facilitation & $\begin{array}{l}\text { Helping patients to talk as fully as } \\
\text { possible about their problems. }\end{array}$ & Yes, I understand. Please continue. \\
\hline & Clarification & $\begin{array}{l}\text { Asking patients to clarify } \\
\text { something they have said. }\end{array}$ & $\begin{array}{l}\text { Please tell me exactly when your } \\
\text { abdominal pain started. }\end{array}$ \\
\hline & Summarizing & $\begin{array}{l}\text { Summarizing the patient's } \\
\text { problems. }\end{array}$ & $\begin{array}{l}\text { You've told me about }- \text {. Is that } \\
\text { right? }\end{array}$ \\
\hline \multirow{5}{*}{$\begin{array}{l}\text { Giving } \\
\text { information }\end{array}$} & $\begin{array}{l}\text { Discovering } \\
\text { the patient's } \\
\text { understanding }\end{array}$ & $\begin{array}{l}\text { Assessing the patient's } \\
\text { understanding of the condition. }\end{array}$ & $\begin{array}{l}\text { Could you tell me what you think is } \\
\text { causing your symptoms? }\end{array}$ \\
\hline & Empathy & $\begin{array}{l}\text { Support by showing an } \\
\text { understanding of the patient's } \\
\text { emotional or physical state. }\end{array}$ & I understand that you are distressed. \\
\hline & Easy terms & $\begin{array}{l}\text { Using short words and short } \\
\text { sentences and avoiding medical } \\
\text { jargon. }\end{array}$ & - \\
\hline & $\begin{array}{l}\text { Checking of } \\
\text { understanding }\end{array}$ & $\begin{array}{l}\text { Checking the patient's } \\
\text { understanding of what has been } \\
\text { said. }\end{array}$ & $\begin{array}{l}\text { Would you like to go over what we } \\
\text { have said? }\end{array}$ \\
\hline & $\begin{array}{l}\text { Giving } \\
\text { opportunities }\end{array}$ & $\begin{array}{l}\text { Giving opportunities for the } \\
\text { patient to ask another questions. }\end{array}$ & Do you have another question? \\
\hline \multirow{5}{*}{$\begin{array}{l}\text { Non- } \\
\text { verbal } \\
\text { communicat } \\
\text { ion }\end{array}$} & Neat feature & Clean appearance. & - \\
\hline & Listening & $\begin{array}{l}\text { Receiving the message and } \\
\text { demonstrating that you are paying } \\
\text { attention and trying to understand. }\end{array}$ & - \\
\hline & Eye contact & Maintaining eye contact properly. & - \\
\hline & Nodding & $\begin{array}{l}\text { Expressing that you understand } \\
\text { by nodding. }\end{array}$ & - \\
\hline & Silence & $\begin{array}{l}\text { Give the patient time to reflect on } \\
\text { what has been said. }\end{array}$ & - \\
\hline
\end{tabular}

\subsection{Data Analysis}

Data processing was performed using the SPSS 14.0 software package. Scores of each item among the communication skills were summarized as mean \pm standard deviation. The correlation between communication skills awareness and performance in the first and second CPX were evaluated with Pearson's correlation analyses. In addition, to identify the improvement of communication skills awareness and performance, the results of each item in the first and second CPX were compared with t-tests. The statistical significance threshold was 0.05 .

\section{Results}

\subsection{Communication Skills Awareness and Performance}

For communication skills awareness in the first CPX, chief complaint score was the highest: $4.94 \pm 0.23$. In addition, greeting, introduction, and listening showed the next highest scores: $4.92 \pm 0.28,4.89 \pm 0.40$, and $4.89 \pm 0.40$, respectively, as shown in Table 2. However, discovering the patient's understanding received the lowest score: $3.31 \pm 1.14$, and empathy, checking of understanding, and facilitation were also relatively lower: $3.42 \pm 1.23,3.58 \pm 1.18$, and $3.92 \pm 0.94$, respectively. 
For communication skills performance in the first CPX, chief complaint showed the highest score: $4.94 \pm 0.23$. In addition, greeting, introduction, and giving opportunities showed the next highest scores: $4.92 \pm 0.28,4.83 \pm 0.51$, and $4.56 \pm 0.84$, respectively. However, discovering the patient's understanding was the lowest score: $2.36 \pm 1.15$, and empathy, checking of understanding, and facilitation were relatively lower: $2.72 \pm$ $1.26,2.75 \pm 1.32$, and $2.89 \pm 0.92$, respectively.

For communication skills awareness in the second CPX, the chief complaint and the giving opportunities scores were the highest: $4.92 \pm 0.37$. Greeting and listening showed the next highest score: $4.89 \pm 0.40$. However, discovering the patient's understanding had the lowest score: $4.03 \pm 1.18$, and facilitation, empathy, and clarification were relatively low: $4.19 \pm 1.06,4.39 \pm 0.90$, and $4.56 \pm 0.88$, respectively.

For communication skills performance in the second CPX, introduction showed the highest score: $4.92 \pm 0.37$. In addition, greeting, chief complaint, and giving opportunities showed the next highest scores: $4.89 \pm 0.40,4.83 \pm 0.51$, and $4.75 \pm 0.55$, respectively. However, discovering the patient's understanding had the lowest score: $2.97 \pm 1.13$, and facilitation, empathy, and silence were relatively low: $3.25 \pm 1.34$, $3.31 \pm 1.17$, and $3.56 \pm 1.05$, respectively.

\subsection{Correlation between Communication Skills Awareness and Performance}

Awareness of greeting $(r=0.273, p=0.108)$ and summarizing $(r=0.251, p=0.140)$ were not correlated with performance in the first CPX, as shown in Table 2.

The other items, including identification $(\mathrm{r}=0.712, \mathrm{p}<0.001)$, introduction $(\mathrm{r}=0.613, \mathrm{p}<$ $0.001)$, attention $(r=0.620, p<0.001)$, chief complaint $(r=1.000, p<0.001)$, open question $(\mathrm{r}=0.496, \mathrm{p}=0.002)$, reflection $(\mathrm{r}=0.591, \mathrm{p}<0.001)$, facilitation $(\mathrm{r}=0.420, \mathrm{p}=0.011)$, clarification $(\mathrm{r}=0.492, \mathrm{p}=0.002)$, discovering the patient's understanding $(\mathrm{r}=0.566, \mathrm{p}<$ $0.001)$, empathy $(r=0.781, p<0.001)$, easy terms $(r=0.489, p=0.002)$, checking of understanding $(\mathrm{r}=0.666, \mathrm{p}<0.001)$, giving opportunities $(\mathrm{r}=0.672, \mathrm{p}<0.001)$, neat features $(\mathrm{r}=0.346, \mathrm{p}=0.039)$, listening $(\mathrm{r}=0.373, \mathrm{p}=0.025)$, eye contact $(\mathrm{r}=0.407, \mathrm{p}=0.014)$, nodding $(\mathrm{r}=0.600, \mathrm{p}<0.001)$, and silence $(\mathrm{r}=0.721, \mathrm{p}<0.001)$ showed significant correlations between awareness and performance of communication skills.

Table 2. Correlation between Communication Skills Awareness and Performance in the First Clinical Performance Examination

\begin{tabular}{|c|c|c|c|c|c|c|c|}
\hline & \multicolumn{2}{|c|}{ Awareness } & \multicolumn{2}{|c|}{ Performance } & \multirow{2}{*}{$\mathrm{r}$} & \multirow{2}{*}{$\mathrm{p}$} \\
\hline & & mean & $\mathrm{SD}$ & mean & $\mathrm{SD}$ & & \\
\hline \multirow{5}{*}{$\begin{array}{l}\text { Beginning } \\
\text { the } \\
\text { interview }\end{array}$} & Greeting & 4.92 & 0.28 & 4.92 & 0.28 & .273 & .108 \\
\hline & Identification & 4.47 & 1.00 & 4.11 & 1.37 & .712 & $<.001$ \\
\hline & Introduction & 4.89 & 0.40 & 4.83 & 0.51 & .613 & $<.001$ \\
\hline & Attention & 4.25 & 0.97 & 3.67 & 1.29 & .620 & $<.001$ \\
\hline & $\begin{array}{l}\text { Chief } \\
\text { complaint }\end{array}$ & 4.94 & 0.23 & 4.94 & 0.23 & 1.000 & $<.001$ \\
\hline \multirow{5}{*}{$\begin{array}{l}\text { Gathering } \\
\text { information }\end{array}$} & Open question & 4.78 & 0.48 & 4.33 & 0.79 & .496 & .002 \\
\hline & Reflection & 4.58 & 0.65 & 3.67 & 1.04 & .591 & $<.001$ \\
\hline & Facilitation & 3.92 & 0.94 & 2.89 & 0.92 & .420 & .011 \\
\hline & Clarification & 4.39 & 0.73 & 3.44 & 0.94 & .492 & .002 \\
\hline & Summarizing & 4.53 & 0.56 & 3.56 & 1.11 & .251 & .140 \\
\hline \multirow{3}{*}{$\begin{array}{c}\text { Giving } \\
\text { information }\end{array}$} & $\begin{array}{l}\text { Discovering } \\
\text { the patient's } \\
\text { understanding }\end{array}$ & 3.31 & 1.14 & 2.36 & 1.15 & .566 & $<.001$ \\
\hline & Empathy & 3.42 & 1.23 & 2.72 & 1.26 & .781 & $<.001$ \\
\hline & Easy terms & 4.44 & 0.73 & 3.69 & 0.79 & .489 & .002 \\
\hline
\end{tabular}




\begin{tabular}{|c|l|c|c|c|c|c|c|}
\hline & $\begin{array}{l}\text { Checking of } \\
\text { understanding }\end{array}$ & 3.58 & 1.18 & 2.75 & 1.32 & .666 & $<.001$ \\
\cline { 2 - 8 } & $\begin{array}{l}\text { Giving } \\
\text { opportunities }\end{array}$ & 4.75 & 0.55 & 4.56 & 0.84 & .672 & $<.001$ \\
\hline \multirow{2}{*}{$\begin{array}{c}\text { Non- } \\
\text { verbal } \\
\text { communicat } \\
\text { ion }\end{array}$} & Neat feature & 4.86 & 0.42 & 4.17 & 0.94 & .346 & .039 \\
\cline { 2 - 8 } & Listening & 4.89 & 0.40 & 4.25 & 0.77 & .373 & .025 \\
\cline { 2 - 8 } & Eye contact & 4.86 & 0.42 & 4.31 & 0.75 & .407 & .014 \\
\cline { 2 - 8 } & Nodding & 4.67 & 0.79 & 3.97 & 0.94 & .600 & $<.001$ \\
\cline { 2 - 8 } & Silence & 4.00 & 1.22 & 3.06 & 1.17 & .721 & $<.001$ \\
\hline
\end{tabular}

In the second CPX, greeting $(r=1.000, p<0.001)$, identification $(r=0.612, p<0.001)$, introduction $(r=0.730, p<0.001)$, attention $(r=0.647, p<0.001)$, chief complaint $(r=0.688$, $p<0.001)$, open question $(r=0.653, p<0.001)$, reflection $(r=0.629, p<0.001)$, facilitation $(r=0.707, p<0.001)$, clarification $(r=0.785, p<0.001)$, summarizing $(r=0.473, p=0.004)$, discovering the patient's understanding $(r=0.661, \mathrm{p}<0.001)$, empathy $(\mathrm{r}=0.697, \mathrm{p}<0.001)$, easy terms $(r=0.465, p=0.004)$, checking of understanding $(r=0.652, p<0.001)$, giving opportunities $(\mathrm{r}=0.595, \mathrm{p}<0.001)$, listening $(\mathrm{r}=0.382, \mathrm{p}=0.021)$, eye contact $(\mathrm{r}=0.388, \mathrm{p}$ $=0.019)$, and nodding $(\mathrm{r}=0.336, \mathrm{p}<0.045)$ showed significant correlations between communication skills awareness and performance.

Both neat feature $(r=0.146, p=0.394)$ and silence $(r=0.272, p=0.109)$ awareness were not correlated with performance (Table 3 ).

Table 3. Correlation between Communication Skills Awareness and Performance in the Second Clinical Performance Examination

\begin{tabular}{|c|c|c|c|c|c|c|c|}
\hline & \multicolumn{2}{|c|}{ Awareness } & \multicolumn{2}{|c|}{ Performance } & \multirow{2}{*}{$\mathrm{r}$} & \multirow{2}{*}{$\mathrm{p}$} \\
\hline & & mean & $\mathrm{SD}$ & mean & SD & & \\
\hline \multirow{5}{*}{$\begin{array}{l}\text { Beginning } \\
\text { the interview }\end{array}$} & Greeting & 4.89 & 0.40 & 4.89 & 0.40 & 1.000 & $<.001$ \\
\hline & Identification & 4.83 & 0.51 & 4.58 & 0.97 & .612 & $<.001$ \\
\hline & Introduction & 4.86 & 0.49 & 4.92 & 0.37 & .730 & $<.001$ \\
\hline & Attention & 4.83 & 0.51 & 4.64 & 0.68 & .647 & $<.001$ \\
\hline & Chief complaint & 4.92 & 0.37 & 4.83 & 0.51 & .688 & $<.001$ \\
\hline \multirow{5}{*}{$\begin{array}{l}\text { Gathering } \\
\text { information }\end{array}$} & Open question & 4.75 & 0.60 & 4.14 & 0.96 & .653 & $<.001$ \\
\hline & Reflection & 4.86 & 0.42 & 4.44 & 0.77 & .629 & $<.001$ \\
\hline & Facilitation & 4.19 & 1.06 & 3.25 & 1.34 & .707 & $<.001$ \\
\hline & Clarification & 4.56 & 0.88 & 3.92 & 1.27 & .785 & $<.001$ \\
\hline & Summarizing & 4.86 & 0.42 & 4.31 & 0.79 & .473 & .004 \\
\hline \multirow{5}{*}{$\begin{array}{c}\text { Giving } \\
\text { information }\end{array}$} & $\begin{array}{l}\text { Discovering } \\
\text { the patient's } \\
\text { understanding }\end{array}$ & 4.03 & 1.18 & 2.97 & 1.13 & .661 & $<.001$ \\
\hline & Empathy & 4.39 & 0.90 & 3.31 & 1.17 & .697 & $<.001$ \\
\hline & Easy terms & 4.72 & 0.57 & 3.97 & 1.06 & .465 & .004 \\
\hline & $\begin{array}{l}\text { Checking of } \\
\text { understanding }\end{array}$ & 4.81 & 0.52 & 4.28 & 0.91 & .652 & $<.001$ \\
\hline & $\begin{array}{l}\text { Giving } \\
\text { opportunities }\end{array}$ & 4.92 & 0.37 & 4.75 & 0.55 & .595 & $<.001$ \\
\hline \multirow{5}{*}{$\begin{array}{c}\text { Non- } \\
\text { verbal } \\
\text { communicatio } \\
n\end{array}$} & Neat feature & 4.83 & 0.51 & 3.89 & 1.28 & .146 & .394 \\
\hline & Listening & 4.89 & 0.40 & 4.36 & 0.83 & .382 & .021 \\
\hline & Eye contact & 4.86 & 0.42 & 4.36 & 0.83 & .388 & .019 \\
\hline & Nodding & 4.83 & 0.45 & 4.06 & 1.01 & .336 & .045 \\
\hline & Silence & 4.58 & 0.73 & 3.56 & 1.05 & .272 & .109 \\
\hline
\end{tabular}




\subsection{Improvement of Communication Skills Awareness and Performance}

Communication skills awareness was significantly improved in the second CPX in terms of attention $(\mathrm{t}=-3.205, \mathrm{p}=0.002)$, reflection $(\mathrm{t}=-2.149, \mathrm{p}=0.036)$, summarizing $(\mathrm{t}=-2.847, \mathrm{p}$ $=0.006)$, discovering the patient's understanding $(\mathrm{t}=-2.636, \mathrm{p}=0.010)$, empathy $(\mathrm{t}=-3.827$, $\mathrm{p}<0.001)$, checking of understanding $(\mathrm{t}=-5.678, \mathrm{p}<0.001)$, and silence $(\mathrm{t}=-2.462, \mathrm{p}=$ 0.017), compared with the first CPX, as shown in Table 4.

In addition, awareness in identification $(\mathrm{t}=-.1 .933 \mathrm{p}=0.059)$, facilitation $(\mathrm{t}=-1.175, \mathrm{p}=$ $0.244)$, clarification $(\mathrm{t}=-0.878, \mathrm{p}=0.383)$, easy terms $(\mathrm{t}=-1.797, \mathrm{p}=0.077)$, giving opportunities $(\mathrm{t}=-1.503, \mathrm{p}=0.138)$, and nodding $(\mathrm{t}=-1.099, \mathrm{p}=0.277)$ showed a tendency toward improvement, but this was not significant.

Table 4. Improvement of Communication Skills Awareness

\begin{tabular}{|c|c|c|c|c|c|c|c|}
\hline & \multicolumn{2}{|c|}{ first CPX } & \multicolumn{2}{|c|}{ second CPX } & \multirow{2}{*}{$\mathrm{t}$} & \multirow{2}{*}{$\mathrm{P}$} \\
\hline & & mean & SD & mean & SD & & \\
\hline \multirow{5}{*}{$\begin{array}{l}\text { Beginning } \\
\text { the interview }\end{array}$} & Greeting & 4.92 & 0.28 & 4.89 & 0.40 & 0.342 & .733 \\
\hline & Identification & 4.47 & 1.00 & 4.83 & 0.51 & -1.933 & .059 \\
\hline & Introduction & 4.89 & 0.40 & 4.86 & 0.49 & 0.265 & .792 \\
\hline & Attention & 4.25 & 0.97 & 4.83 & 0.51 & -3.205 & .002 \\
\hline & $\begin{array}{l}\text { Chief } \\
\text { complaint }\end{array}$ & 4.94 & 0.23 & 4.92 & 0.37 & 0.383 & .703 \\
\hline \multirow{5}{*}{$\begin{array}{l}\text { Gathering } \\
\text { information }\end{array}$} & Open question & 4.78 & 0.48 & 4.75 & 0.60 & 0.215 & .830 \\
\hline & Reflection & 4.58 & 0.65 & 4.86 & 0.42 & -2.149 & .036 \\
\hline & Facilitation & 3.92 & 0.94 & 4.19 & 1.06 & -1.175 & .244 \\
\hline & Clarification & 4.39 & 0.73 & 4.56 & 0.88 & -0.878 & .383 \\
\hline & Summarizing & 4.53 & 0.56 & 4.86 & 0.42 & -2.847 & .006 \\
\hline \multirow{5}{*}{$\begin{array}{l}\text { Giving } \\
\text { information }\end{array}$} & $\begin{array}{l}\text { Discovering } \\
\text { the patient's } \\
\text { understanding }\end{array}$ & 3.31 & 1.14 & 4.03 & 1.18 & -2.636 & .010 \\
\hline & Empathy & 3.42 & 1.23 & 4.39 & 0.90 & -3.827 & $<.001$ \\
\hline & Easy terms & 4.44 & 0.73 & 4.72 & 0.57 & -1.797 & .077 \\
\hline & $\begin{array}{l}\text { Checking of } \\
\text { understanding }\end{array}$ & 3.58 & 1.18 & 4.81 & 0.52 & -5.678 & $<.001$ \\
\hline & $\begin{array}{l}\text { Giving } \\
\text { opportunities }\end{array}$ & 4.75 & 0.55 & 4.92 & 0.37 & -1.503 & .138 \\
\hline \multirow{5}{*}{$\begin{array}{c}\text { Non- } \\
\text { verbal } \\
\text { communicatio } \\
n\end{array}$} & Neat feature & 4.86 & 0.42 & 4.83 & 0.51 & 0.252 & .802 \\
\hline & Listening & 4.89 & 0.40 & 4.89 & 0.40 & 0.000 & 1.000 \\
\hline & Eye contact & 4.86 & 0.42 & 4.86 & 0.42 & 0.000 & 1.000 \\
\hline & Nodding & 4.67 & 0.79 & 4.83 & 0.45 & -1.099 & .277 \\
\hline & Silence & 4.00 & 1.22 & 4.58 & 0.73 & -2.462 & .017 \\
\hline
\end{tabular}

Communication skills performance was significantly improved in terms of attention $(\mathrm{t}=$ 4.004, $\mathrm{p}<0.001)$, reflection $(\mathrm{t}=-3.598, \mathrm{p}=0.001)$, summarizing $(\mathrm{t}=-3.314, \mathrm{p}=0.001)$, discovering the patient's understanding $(\mathrm{t}=-2.271, \mathrm{p}=0.026)$, empathy $(\mathrm{t}=-2.042, \mathrm{p}=$ $0.045)$, and checking of understanding $(\mathrm{t}=-5.717, \mathrm{p}<0.001)$.

In addition, performance in identification $(\mathrm{t}=-1.691, \mathrm{p}=0.096)$, introduction $(\mathrm{t}=-0.798, \mathrm{p}$ $=0.428)$, facilitation $(\mathrm{t}=-1.334, \mathrm{p}=0.187)$, clarification $(\mathrm{t}=-1.791, \mathrm{p}=0.078)$, giving opportunities $(\mathrm{t}=-1.156, \mathrm{p}=0.252)$, listening $(\mathrm{t}=-0.588, \mathrm{p}=0.559)$, eye contact $(\mathrm{t}=-0.297$, $\mathrm{p}=0.767)$, nodding $(\mathrm{t}=-0.362, \mathrm{p}=0.719)$, and silence $(\mathrm{t}=-1.905, \mathrm{p}=0.061)$ tended to be improved, but these effects were not significant (Table 5). 
Table 5. Improvement of Communication Skills Performance

\begin{tabular}{|c|c|c|c|c|c|c|c|}
\hline & \multicolumn{2}{|c|}{ first CPX } & \multicolumn{2}{|c|}{ second CPX } & \multirow{2}{*}{$\mathrm{t}$} & \multirow{2}{*}{$\mathrm{P}$} \\
\hline & & mean & SD & mean & SD & & \\
\hline \multirow{5}{*}{$\begin{array}{l}\text { Beginning } \\
\text { the interview }\end{array}$} & Greeting & 4.92 & 0.28 & 4.89 & 0.40 & 0.342 & .733 \\
\hline & Identification & 4.11 & 1.37 & 4.58 & 0.97 & -1.691 & .096 \\
\hline & Introduction & 4.83 & 0.51 & 4.92 & 0.37 & -0.798 & .428 \\
\hline & Attention & 3.67 & 1.29 & 4.64 & 0.68 & -4.004 & $<.001$ \\
\hline & $\begin{array}{l}\text { Chief } \\
\text { complaint }\end{array}$ & 4.94 & 0.23 & 4.83 & 0.51 & 1.195 & .238 \\
\hline \multirow{5}{*}{$\begin{array}{l}\text { Gathering } \\
\text { information }\end{array}$} & Open question & 4.33 & 0.79 & 4.14 & 0.96 & 0.937 & .352 \\
\hline & Reflection & 3.67 & 1.04 & 4.44 & 0.77 & -3.598 & .001 \\
\hline & Facilitation & 2.89 & 0.92 & 3.25 & 1.34 & -1.334 & .187 \\
\hline & Clarification & 3.44 & 0.94 & 3.92 & 1.27 & -1.791 & .078 \\
\hline & Summarizing & 3.56 & 1.11 & 4.31 & 0.79 & -3.314 & .001 \\
\hline \multirow{5}{*}{$\begin{array}{c}\text { Giving } \\
\text { information }\end{array}$} & $\begin{array}{l}\text { Discovering } \\
\text { the patient's } \\
\text { understanding }\end{array}$ & 2.36 & 1.15 & 2.97 & 1.13 & -2.271 & .026 \\
\hline & Empathy & 2.72 & 1.26 & 3.31 & 1.17 & -2.042 & .045 \\
\hline & Easy terms & 3.69 & 0.79 & 3.97 & 1.06 & -1.267 & .210 \\
\hline & $\begin{array}{l}\text { Checking of } \\
\text { understanding }\end{array}$ & 2.75 & 1.32 & 4.28 & 0.91 & -5.717 & $<.001$ \\
\hline & $\begin{array}{l}\text { Giving } \\
\text { opportunities }\end{array}$ & 4.56 & 0.84 & 4.75 & 0.55 & -1.156 & .252 \\
\hline \multirow{5}{*}{$\begin{array}{c}\text { Non- } \\
\text { verbal } \\
\text { communicati } \\
\text { on }\end{array}$} & Neat feature & 4.17 & 0.94 & 3.89 & 1.28 & 1.048 & .298 \\
\hline & Listening & 4.25 & 0.77 & 4.36 & 0.83 & -0.588 & .559 \\
\hline & Eye contact & 4.31 & 0.75 & 4.36 & 0.83 & -0.297 & .767 \\
\hline & Nodding & 3.97 & 0.94 & 4.06 & 1.01 & -0.362 & .719 \\
\hline & Silence & 3.06 & 1.17 & 3.56 & 1.05 & -1.905 & .061 \\
\hline
\end{tabular}

\section{Discussion}

Inappropriate communication with patients can lead to negative prognosis because distorted information about patients can cause the physician to make incorrect decisions regarding treatment plans and may generate overall negative responses to patient care. Especially in patients with cancer or severe diseases, with whom healthcare providers have an especially hard time generating helpful interpersonal relationships with the usual communication skills, adapting to proper medical communication skills can make healthcare providers more aware and understanding of a patient's state, enabling them to provide comforting empathy to their patients and to perform harmonious patient care.

Medical communication usually consists of the phases of beginning an interview, gathering information, and giving information, accompanied by non-verbal communication in the total communication process. Communication skills at the beginning of an interview include greeting, identification, introduction, and attention. The communication skills of gathering information include open and closed questions, reflection, facilitation, clarification, summarizing, and silence. The communication skills of giving information include finding out what the patient thinks, expressing empathy to the patient, explanation medical situations with easy terms, checking for patient's understanding, and giving opportunities. In addition, the non-verbal communication skills include maintaining neat features, proper listening, adequate eye contact, and appropriate nodding [4]. 
We performed communication skills assessments during two clinical performance examinations of medical students. The Objective Structured Clinical Examination was the most commonly used method of assessment, and the average number of assessments per year was 2.4 in UK medical schools [5].

Clinical education focusing on medical communication seems to have positive effects in most medical and nursing schools. In a systematic review of 47 studies on communication training for oncology health professionals, all the interventions demonstrated modest improvements in communication skills, and positive attitudes and beliefs help maintain skills over time in clinical practice and aid in the effective handling of emotional situations [6]. In addition, students' communication skills were improved with more practice and longer training, especially with regard to opening the interview, expressing empathy, understanding the patient's perspective, and preparing for the physical examination in another study of medical students who have completed their third year clerkship [7].

This study also showed that clinical education of communication skills improved communication skills awareness and performance in attention, reflection, summarizing, discovering the patient's understanding, enhancing empathy, checking understanding, and silence after an eight-month clerkship. This educational effect of communication skill can be maintained for long periods of time for some items. One study in which the correlation between medical students' seven communication and interpersonal skills test scores and the scores of these same individuals during their residencies three years later showed the significant correlation for explaining more explicably [8].

However, the effects of education in communication skills differed substantially across situations and was questionable whether communication skills education benefits could be transferred to actual medical communication with patients in order to make meaningful outcomes. One study performed to identify the correlation between communication skills for emotional empathy and academic achievement showed that male students have higher scores than females for all communication skills except verbal expression empathy, and that the correlation between communication skills and academic achievement varied greatly according to clinical performance examination subjects [9]. In addition, among internal medicine residents and nurse practitioner trainees, simulation-based communication training, compared with usual education, did not improve communication quality and patient care, and was associated with a small increase in patients' depressive symptoms [3].

The significant correlation between communication skills awareness and performance suggests that awareness improvement through communication skills education could also improve medical student communication performance [10]. In this study, both communication skills awareness and performance were improved after eight months of clinical education.

However, it is necessary to clarify whether communication skills education can improve communication with real patients in the future. Communication is a powerful therapeutic tool and nursing skill necessary for reaching positive health goals. Education that involves only the transfer of knowledge not only is ineffective but also fails to invoke the conditions whereby this knowledge is converted into a skill [11]. Although patients highly rated medical graduates' communication skills, patients' ages and educational levels were significantly associated with the rating level. Patients who were older than 45 years gave higher scores than did younger ones, and patients with higher education reported much lower scores than did those with less education [12].

Therefore, it is necessary to make an effort to find effective methods of teaching communication skills in a clinical situation, considering the effect of communication skills education and patients' assessment. 


\section{Conclusion}

This study demonstrated that medical students' awareness of communication skills was correlated significantly with performance. In addition, communication awareness and performance were improved following clinical education.

\section{References}

[1] W. Levinson, C. S. Lesser and R. M. Epstein, "Developing Physician Communication Skills For PatientCentered Care", Health Affairs, vol. 29, no. 7, (2010), pp. 1310-1317.

[2] M. Kraft and G. Neitzke, "Communication in medical education: Students' demands", Medicine, Health Care and Philosophy, vol. 3, (2000), pp. 185-190.

[3] J. R. Curtis, A. L. Back, D. W. Ford, L. Downey, S. E. Shannon, A. Z. Doorenbos, E. K. Kross, L. F. Reinke, L. C. Feemster, B. Edlund, R. W. Arnold, O. Kim and R. A. Engelberg, "Effect of Communication Skills Training for Residents and Nurse Practitioners on Quality of Communication With Patients With Serious Illness. A Randomized Trial”, Journal of American Medical Association, vol. 310, no. 21, (2013), pp. 22712281.

[4] M. Lloyd and R. Bor, "Communication Skills For Medicine", Churchill Livingstone Elsevier, London (2009).

[5] A. Laidlaw, H. Salisbury, E. M. Doherty and C. Wiskin, "National survey of clinical communication assessment in medical education in the United Kingdom (UK)", BMC Medical Education, vol. 14, no. 10, (2014).

[6] M. Gysels, A. Richardson and I. J. Higginson, "Communication training for health professionals who care for patients with cancer: a systematic review of effectiveness", Support Care Cancer, vol. 12, no. 12, (2004), pp. 692-700.

[7] Y. H. Kim, J. H. Yang, S. Y. Ahn, S. Y. Song and H. R. Roh, "Communication Skills Improvement of Medical Students According to Length and Methods of Preclinical Training”, Korean Journal of Medical Education, vol. 21, no. 1, (2009), pp. 3-16.

[8] W. B. Park, S. R. Phyo, E. Y. Jang, S. H. Kang, S. J. Myung, H. Y. Shin, Y. S. Lee and J. S. Shin, "Correlations of Communication and Interpersonal Skills between Medical Students and Residents", Korean Journal of Medical Education, vol. 22, no. 4, (2010), pp. 269-274.

[9] S. S. Jang, J. H. Seo, G. J. Cho, S. C. Hong and H. O. Woo, "Correlation of Communication Skills for Emotional Empathy and Academic Achievement on Clinical Performance Examinations", Korean Journal of Medical Education, vol. 22, no. 2, (2010), pp. 121-130.

[10] W. J. Kim and M. Y. Kim, "Awareness and Performance of Communication Skills in Clinical Education", Proceedings of Advanced Science and Technology Letters ASTL 47, Jeju, Korea, (2014) April 15-18.

[11] B. Üstün, "Communication Skills Training as Part of a Problem-Based Learning Curriculum", Journal of Nursing Education, vol. 45, no. 10, (2006), pp. 421-424.

[12] F. T. Abadel and A. S. Hattab, "Patients' assessment of professionalism and communication skills of medical graduates”, BMC Medical Education, vol. 14, no. 28, (2014).

\section{Authors}

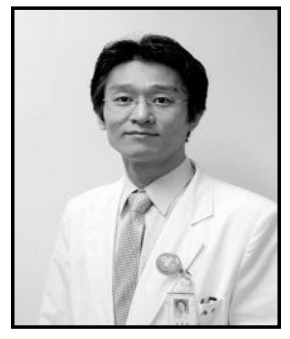

\section{Woo Jeong Kim}

Aug. 2007 : Seoul National University PhD.

Mar. 2005 - current : associate professor, Jeju National University

Research Interest : emergency medicine, medical education

E-mail : gurum21@jejunu.ac.kr

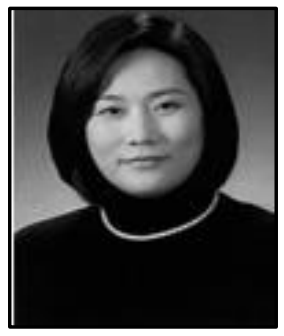

\section{Min Young Kim}

Aug. 2008 : Seoul National University PhD.

Mar. 2011 - current : assistant professor, Jeju National University

Research Interest : nursing education, oncology nursing, nursing management

E-mail : musemy2@jejunu.ac.kr 
International Journal of Bio-Science and Bio-Technology Vol.6, No.4 (2014) 